\title{
Quality Inspection and Classification of Mangoes using Color and Size Features
}

\author{
Ankur M Vyas \\ Computer Engineering Dept. \\ Sardar Vallabhbhai Patel \\ Institute of technology, \\ Vasad, Gujarat, India
}

\author{
Bijal Talati \\ HOD, Computer Engineering \\ Dept., Sardar Vallabhbhai Patel \\ Institute of technology, \\ Vasad, Gujarat, India
}

\author{
Sapan Naik \\ Asst. Prof. Computer Science \\ and Tech. Dept., \\ Uka Tarsadia University, \\ Bardoli, Gujarat, India
}

\begin{abstract}
In terms of the productions of fruits and vegetables India holds the $2^{\text {nd }}$ rank after China. India lacks of the automatic grading system even though the production of the agricultural products is so high. In India most of the grading of fruits are based on size feature which is done manually. Color and size are the most vital parameters to inspect the quality of the fruits.

So the objective of this paper is to develop an algorithm for the automated grading system of mangoes which would be economically beneficial to the agriculture. The dataset of the mangoes are collected in Unripe, Semi Ripe and Ripe phases. They are given as the input to the system for processing. From the image of the mango the color and size feature extraction takes place. The dominant intensity of the ' $a$ ' channel of the Lab color space accounts for the color feature of the mango. The length of the major axis accounts for the size feature. Based on the extracted parameters with grading rules, the mango is classified into grade1, grade2, grade 3 or rejected. Results show that the grading accuracy of $94.97 \%$ was observed
\end{abstract}

\section{General Terms}

Quality Inspection, Feature Extraction, Color Feature.

\section{Keywords}

Mango Grading, Grading System, Image Processing, Color Feature Extraction

\section{INTRODUCTION}

India ranks 2 nd in terms of the production of the fruits and vegetables next to China. Even though having such a high rank in terms of production, India lacks of the scientific automatic grading systems. Currently, grading of the agricultural commodities in the country is done by the human experts based on its visual characteristics. The development of the automatic grading systems can improve the revenue of these commodities, as a result saving time as well as human labor. Image processing can play a vital part in this automation. Color, size and shape parameters are extracted from the image of the fruits and can be taken as the feature parameters for the grading.

The automated strawberry grading system was presented in [1]. In that color, shape and size parameters were used for the grading. Dominant color feature extraction technique was used for the color and largest diameter was taken into account for the size. Mango grading system was introduced in [2]. Color, skin and size feature was used in grading of the mangoes. Fuzzy rules were created for the grading. An accuracy of over $80 \%$ was observed. Another grading system of the mangoes using the fuzzy logic was observed in [3]. The length, height and width of the mango were given to the fuzzy grading system. Grading of the apples using the neural network using the color as feature parameter was presented in [4]. Lemon sorting system [5] based on the color and size as the feature parameters was also developed. HIS color model was used. Because the Hue value of HIS is stable this color model was used in lemon sorting.

In the apple grading system [6] the novel image segmentation technique was proposed for the color images that used Otsu threshold method and support vector machine (SVM). This method was used to sort the apple into different grades. SVM was used for classification of apples which take less training time and also provide better results. This paper presented the grading the oil fresh fruit bunches [7] into over ripe, ripe and unripe on the basis of the color feature extraction.

The date fruit grading system was developed in [8]. The features such as flabbiness, size, shape, intensity and defects were extracted from date. RGB images of the date were used and grading was done on the basis of extracted features in to grades 1, 2 and 3. Accuracy of $80 \%$ was observed. The citrus grading system was presented in [9] and the orange grading system was developed in [10].Summarizing all the automatic grading system, it was found that majority of the grading systems takes color and size as their feature parameters for the grading the fruits into various grades.

The objective of this paper is to develop an algorithm for the automated grading system of mangoes which would be economically beneficial to the agriculture. Color and size of the mango will be taken as the feature parameters for the grading them into grade1, grade2, grade 3 and rejected.

\section{MATERIALS AND METHODS}

\subsection{Collection of Mango Samples}

Two varieties of mangoes namely Totapuri and Badami were used for the experimentation purpose. They are very popular in the region so they were selected for the experimentation purpose. The images were captured in three phase namely unripe, semi ripe and ripe phase. The collection of samples was from the Navsari (Gujarat), from a mango farm. The image dataset contains nearly more than 50 samples for each grade category of the mangoes. Some sample images are shown in Fig 1. The verification of the grading of the mangoes that a mango classifies in to a grade is done by an expert farmer having a very rich experience of 25 years. 


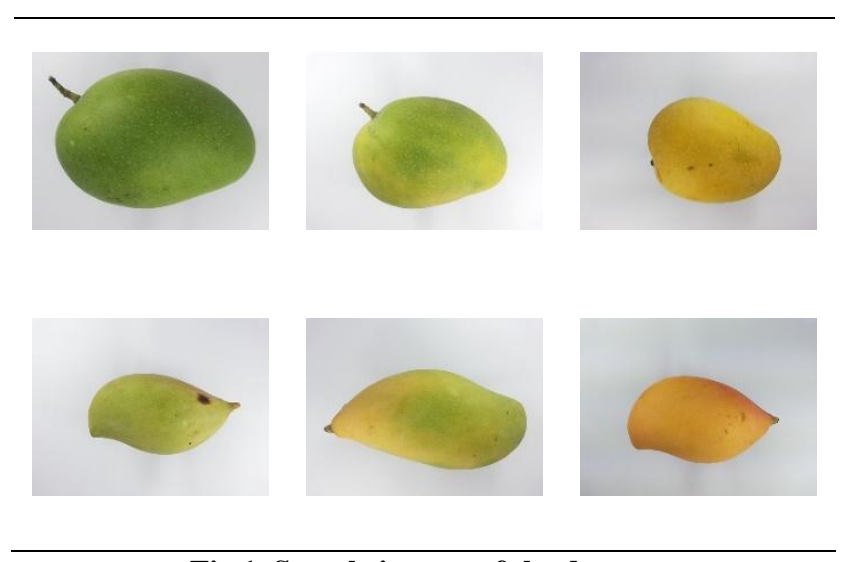

Fig 1. Sample images of the dataset

\subsection{Image Acquisition and Pre-processing Operations}

The collection of samples was using the camera (Nikon DSLR) in the image acquisition chamber. The Lamp was used in the in order to provide ambient lightning conditions and to correctly capture the surface of the mango. To reduce shadow effect the chamber was coated with the reflective material. About 400 images in total were captured. The mechanism for the image capturing is shown in Fig2.

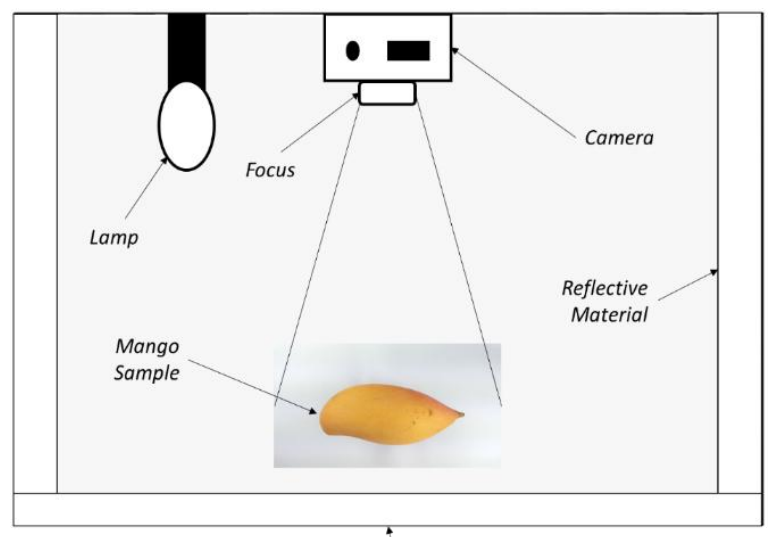

Image Acquisition Chamber

Fig 2. Set up for Image Acquisition

Matlab was used for the experimentation purpose as it is a powerful tool for the Image Processing. An image was then given as input for the processing as per Fig 4. Then conversion to the grayscale image takes place as in Fig 5 . Simple 3X3 median filter was used to remove the noise from the image. Median filter was used because it removes the noise from the image and also preserves the edge information in the image. Otsu thresholding was used to segment the mango from the background. As a result the binary image was obtained as per Fig 6. First erosion and then dilation operations were performed in order to smooth the curvature of the mango. This resultant image was used as a mask in the original RGB image for the background subtraction. And we get the resultant image consisting of segmented mango without the background pixels. The flowchart of whole grading process is shown in Fig 3.

After the pre-processing stage is completed the whole segmented mango is taken and spots pixels are extracted. The black and the brown pixels coming inside the area of the mango are taken as the spot pixels. The ratio of the spots pixels to the pixels of the mango as Eq.(1) are taken and from that the spots ratio value the categorization into less, average and more spots for a mango takes place.
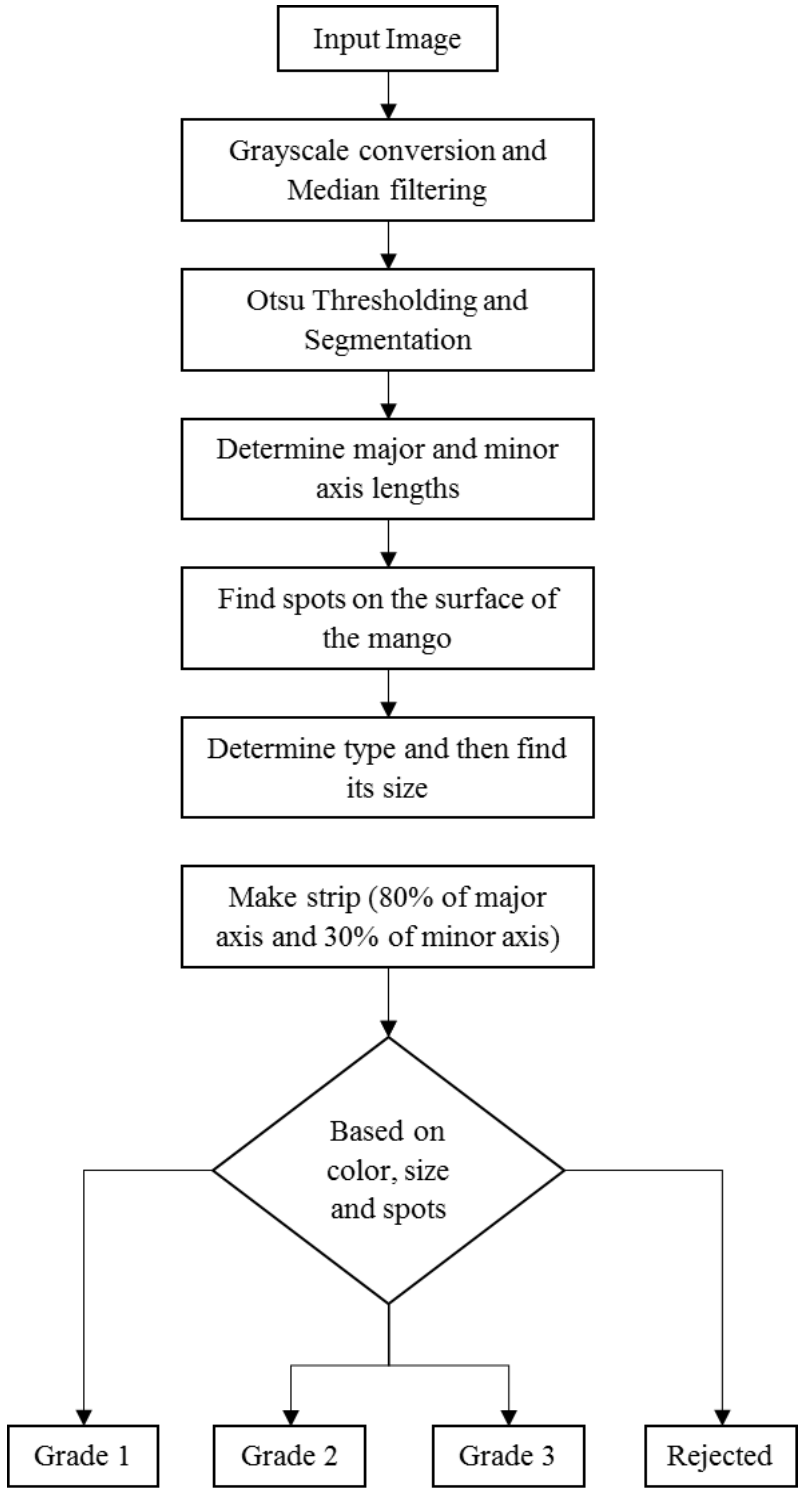

Fig 3. Flowchart of whole grading process

Spots Ratio $=\frac{\text { Spots Pixels }}{\text { Total Mango Pixels }}$

After that the major and minor axis length are extracted from the segmented mango. The length are converted into inches by dividing by the dpi (dots per inches) of the image. Totapuri mangoes are having the oblong shape i.e. the length is more as compared to the width. So we can easily classify the Badami and Totapuri mangoes on the basis of their ratio of major and minor axis lengths as per Eq.(2). If this ratio is more than 1.5 then it is Totapuri and if less than 1.5 then it is categorized as Badami.

Ratio $=\frac{\text { Major Axis Length }}{\text { Minor Axis Length }}$

Now as the type categorization completes then on the basis of the major axis length the size is given. The Totapuri mangoes are larger in size and Badami are smaller on an average. So different ranges have been found out for the categorization of mangoes into small, medium or large sizes. 


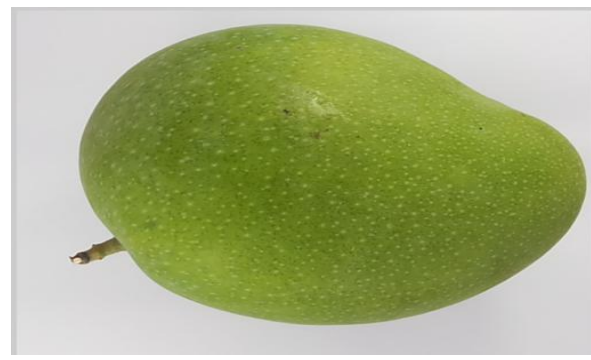

Fig 4. Input Mango image

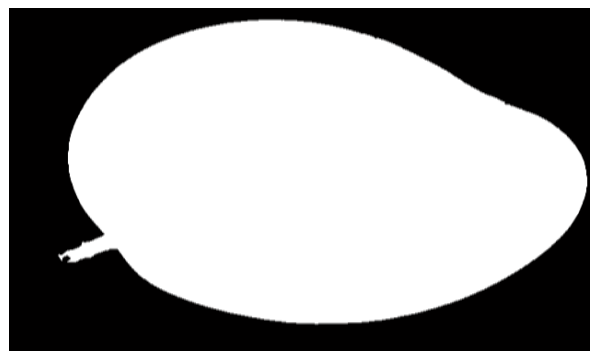

Fig 6. Image after thresholding

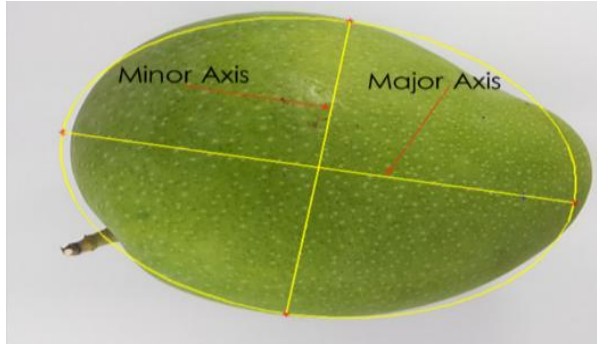

Fig 8.Major and Minor axis lengths

\subsection{Color Feature Extraction}

Color is the most vital feature for the grading of the mangoes. Different color feature extraction techniques were presented in [11]. No color model is superior than another still because of the RGB color model is sensitive to the lightning conditions it is generally a poor choice for the color feature extraction process. Here the Lab color model was used for this purpose[13]. As the images are in the RGB color space when it was captured from the camera so it need to be transformed into the Lab color space. Direct conversion to Lab is not possible so the RGB coordinates are first transformed in to $\mathrm{XYZ}$ and then to Lab coordinates using below equations as in [12].

$\left[\begin{array}{l}X \\ Y \\ Z\end{array}\right]=\left[\begin{array}{ccc}0.607 & 0.174 & 0.200 \\ 0.299 & 0.587 & 0.114 \\ 0.00 & 0.066 & 1.116\end{array}\right]\left[\begin{array}{l}R \\ G \\ B\end{array}\right]$

$L^{*}=116 *\left(\sqrt[3]{\frac{Y}{Y_{0}}}\right)-16$

$a^{*}=500 *\left[\sqrt[3]{\frac{X}{X_{0}}}-\sqrt[3]{\frac{Y}{Y_{0}}}\right]$

$b^{*}=200 *\left[\sqrt[3]{\frac{Y}{Y_{0}}}-\sqrt[3]{\frac{Z}{Z_{0}}}\right]$

Where $\frac{X}{X_{0}}>0.01, \frac{Y}{Y_{0}}>0.01$ and $\frac{Z}{Z_{0}}>0.01 .\left(X_{0}, Y_{0}, Z_{0}\right)$ shows $\mathrm{X}, \mathrm{Y}$ and $\mathrm{Z}$ values of standard white.

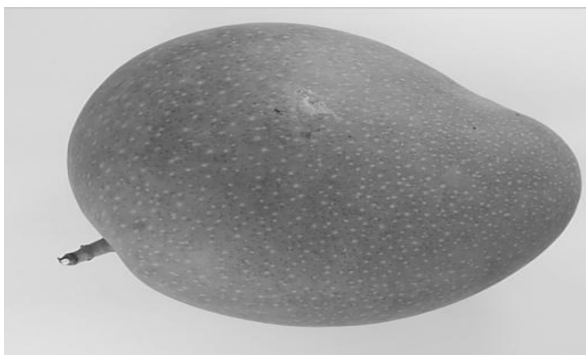

Fig 5. Conversion to grayscale

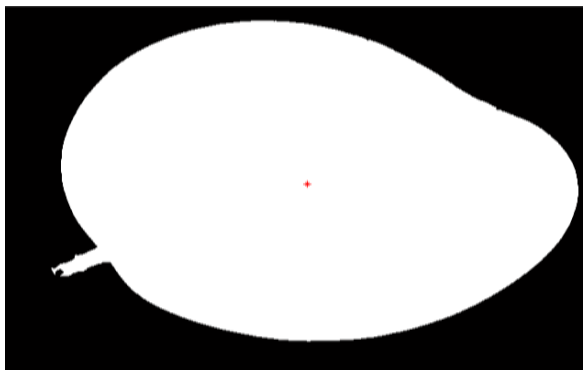

Fig 7. Centroid of mango (red *)

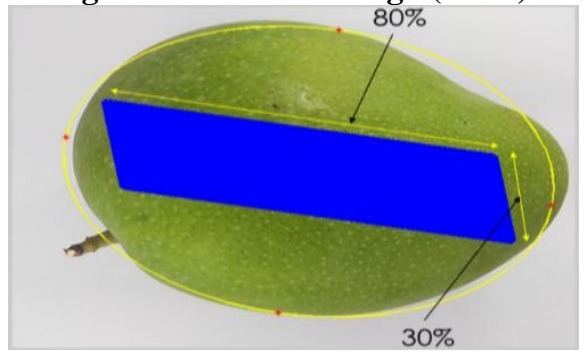

Fig 9. Strip of the mango

Now a modification has been proposed here. Instead of converting the whole image into the Lab color space coordinates the strip as shown in Fig 9 is only converted into the Lab color space. Instead of creating the histogram of the whole mango only the histogram of the strip is created. By doing that time for the conversion and processing which is so very crucial in grading is saved.

The process of making the strip starts by calculating the centroid of the mango as per Fig 7. And then taking $80 \%$ area on the major axis and $30 \%$ area on the minor axis as the strip as shown in Fig 9. Histogram analysis on the strip is then done.

\section{Strip Histogram Analysis}

The dominant color feature extraction technique has been explained in [1]. In that the histograms over the 'a' channel of the Lab color model was created. The window of certain length was chosen and averaging of the window values was done. Then the window having the maximum value was chosen as the dominant color. The color calculated by this technique is very close to human vision so this technique is very effective.

A modification in this technique is proposed instead to taking the window, the bin having the maximum pixels is chosen as the dominant bin as per Fig 10. So this modified technique for the histogram analysis was adopted on the strip of the mango. 
After the analysis it was found that categorization into unripe, semi ripe and ripe is possible by the range as in Table 1 .

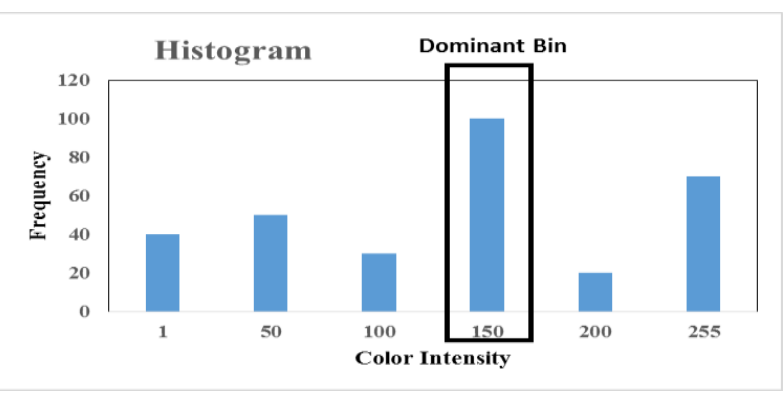

Fig 10. Dominant bin in histogram

The following range of the dominant bin over ' $a$ ' channel of Lab color space was observed

Table 1: Dominant 'a' color range

\begin{tabular}{cc}
\hline Dominant a & Category \\
\hline$a<=117$ & Unripe Mango \\
$117<a<130$ & Semi Ripe Mango \\
$a>=130$ & Ripe Mango \\
\hline
\end{tabular}

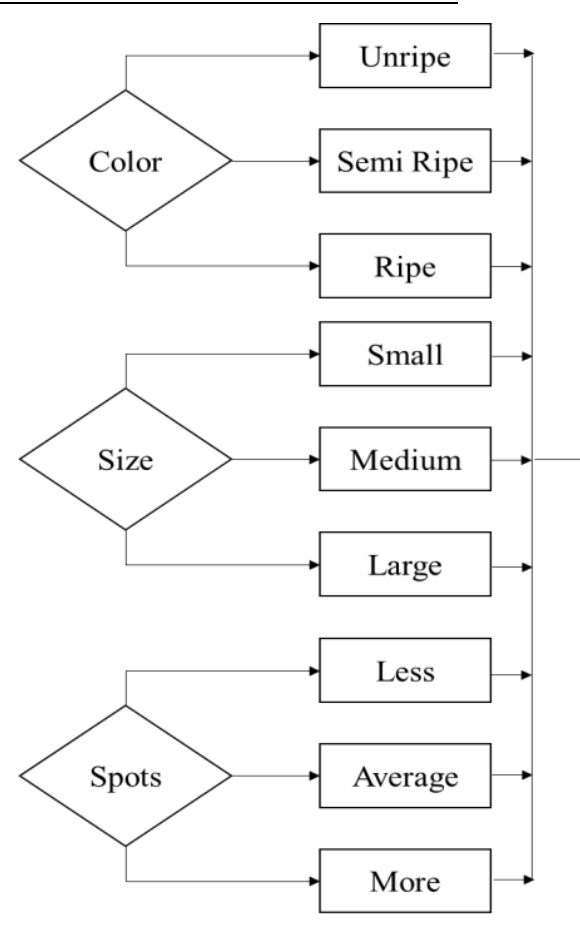

On the basis of grading rules results are shown in section 3 .

So at this point the dominant color, spots and size features are extracted from the mango. All the features with corresponding values are shown in Fig 11. These are the feature parameters for the grading. Based on this the grading rules are created. Some of them are shown Table 2 .

Table 2: Sample rules of grading

\section{Rule 1}

If spots are more and Size is less and

Color is unripe then Grade is Rejected

\section{Rule 8}

If spots are less and

Size is medium and

Color is semi ripe then Grade is Grade 2

\section{Rule 4}

If spots are average and Size is medium and Color is unripe then Grade is Grade 3

\section{Rule 10}

If spots are less and Size is large

Color is Ripe then Grade is Grade 1

Fig11. Feature parameters and values

\section{RESULTS AND DISCUSSION}

After the implementation of the algorithm, comparison of the two methods have been shown here. First is by taking the full mango for the color feature extraction as in Table 3. Second is the result by taking only the strip for the color feature extraction as in Table 4 . Rest of the steps are same. Time taken for the grading is shown in Table 5 in seconds. Proposed method does the grading of a mango in less than a second.
Table 3. Accuracy of using full mango

\begin{tabular}{|c|c|c|c|}
\hline Grade & $\begin{array}{c}\text { Correctly } \\
\text { Classified } \\
\text { Samples }\end{array}$ & $\begin{array}{c}\text { Total } \\
\text { Correct } \\
\text { Samples }\end{array}$ & $\begin{array}{c}\text { Accuracy } \\
\text { Full } \\
\text { Mango }\end{array}$ \\
\hline Extra Class & 86 & 86 & $100 \%$ \\
\hline Grade 1 & 51 & 58 & $87.93 \%$ \\
\hline Grade 2 & 166 & 196 & $84.69 \%$ \\
\hline Rejected & 52 & 58 & $89.66 \%$ \\
\hline \multicolumn{3}{|c|}{ Average Accuracy } & $\mathbf{9 0 . 5 7 \%}$ \\
\hline
\end{tabular}


Table 4. Accuracy with the proposed method

\begin{tabular}{|c|c|c|c|}
\hline Grade & $\begin{array}{c}\text { Correctly } \\
\text { Classified } \\
\text { Samples }\end{array}$ & $\begin{array}{c}\text { Total } \\
\text { Correct } \\
\text { Samples }\end{array}$ & $\begin{array}{c}\text { Accuracy } \\
\text { Proposed } \\
\text { Method }\end{array}$ \\
\hline Extra Class & 86 & 86 & $100 \%$ \\
\hline Grade 1 & 55 & 58 & $94.82 \%$ \\
\hline Grade 2 & 187 & 196 & $95.40 \%$ \\
\hline Rejected & 52 & 58 & $89.66 \%$ \\
\hline \multicolumn{3}{|c|}{ Average Accuracy } & $\mathbf{9 4 . 9 7 \%}$ \\
\hline
\end{tabular}

Table 5. Comparison of time

\begin{tabular}{|c|c|c|}
\hline \multicolumn{1}{|c|}{ Full Mango } & Proposed Method \\
\hline Time (seconds) & $499.43 \mathrm{secs}$ & $377.23 \mathrm{secs}$ \\
\hline $\begin{array}{c}\text { Average time for } \\
\text { 1 mango }\end{array}$ & $1.248 \mathrm{sec}$ & $0.9425 \mathrm{sec}$ \\
\hline
\end{tabular}

\section{CONCLUSION AND FUTURE DIRECTION}

An algorithm for the grading of the mangoes was developed. Color, spots and size were taken as the feature parameters for the grading the mangoes into grade1, grade2, grade 3 and rejected. A modified dominant color feature extraction technique using the Lab color model had been proposed. Instead of taking the whole mango the color feature was extracted from the strip on the surface of mango. The spots were extracted from the whole surface of the mango. Length of the major axis was used for the size feature. Experiment shows that the proposed method has more accuracy as well as efficiency.

Proposed algorithm was able to classify the mangoes with an accuracy of $94.97 \%$ into various grades. And time taken for the grading of a mango on average was less than a second. So the proposed method is accurate as well as efficient. In the future work the texture on the surface of the mango can also be used as a feature parameter for grading so that the overall accuracy can be improved.

\section{Acknowledgement}

Authors would like to thank Mr.Ruknuddin Kazi (Valsad, Gujarat) for the authentication of the dataset of the mangoes.

\section{REFERENCES}

[1] Xu Liming and Zhao Yanchao, "Automated strawberry grading system based on image processing," Computers and Electronics in Agriculture, vol. 71, no. Supplement 1, pp. S32-S39, April 2010.

[2] Tajul Rosli Bin Razak, Mahmod Bin Othman(DR), Mohd Nazari Bin Abu Bakar(DR), Khairul Adilah BT
Ahmad, and AB.Razak Bin Mansor, "Mango Grading By Using Fuzzy Image Analysis," in In proceedings of International Conference on Agricultural, Environment and Biological Sciences, Phuket, 2012.

[3] Teoh, Yeong Kin, Abu Hasan, Suzanawati, AND Sauddin@Sa'duddin, Suraiya. "Automated Mango Fruit Grading System Using Fuzzy Logic" Journal of Agricultural Science [Online], Volume 6 Number 1, December 2013.

[4] Kazuhiro Nakano, "Application of neural networks to the color grading of apples," Computers and Electronics in Agriculture, vol. 18, no. 2-3, pp. 105-116, August 1997.

[5] M. Khojastehnazhand, M. Omid, and A. Tabatabaeefar, "Development of a lemon sorting system based on color and size," African Journal of Plant Science, vol. 4(4), pp. 122-127, April 2010.

[6] Akira Mizushima and Renfu Lu, "An image segmentation method for apple sorting and grading using support vector machine and Otsu's method," Computers and Electronics in Agriculture, vol. 94, pp. 29-37, June 2013.

[7] Nursuriati Jamil, Azlinah Mohamed, and Syazwani Abdullah, "Automated Grading of Palm Oil Fresh Fruit Bunches (FFB) using Neuro-Fuzzy Technique," 2009 International Conference of Soft Computing and Pattern Recognition, pp. 245-249, 2009.

[8] Yousef Al Ohali, "Computer vision based date fruit grading system: Design and implementation," Journal of King Saud University - Computer and Information Sciences, vol. 23, no. 1, pp. 29-39, January 2011

[9] U. Ahmad, M. Suhil, R. Tjahjohutomo, and H.K. Purwadaria, "Development of Citrus Grading System Using Image Processing". [Online] http://repository.ipb.ac.id/handle/123456789/68 910

[10] Naoshi Kondo, Usman Ahmad, Mitsuji Monta, and Haruhiko Murase, "Machine vision based quality evaluation of Iyokan orange fruit using neural networks," Computers and Electronics in Agriculture, vol. 29, no. 12, pp. 135-147, October 2000

[11] Ankur M Vyas, Bjial Talati and Sapan Naik. Article: Colour Feature Extraction Techniques of Fruits: A Survey. International Journal of Computer Applications 83(15):15-22, December 2013. Published by Foundation of Computer Science, New York, USA.

[12] Cheng, Heng-Da, X. H. Jiang, Ying Sun, and Jingli Wang. "Colour image segmentation: advances and prospects." Pattern recognition 34, no. 12 (2001): 22592281.

[13] Naik S. and Patel B, "CIELab based color feature extraction for maturity level grading of Mango (Mangifera Indica L ), National journal of System and Information technology, ISSN: 09743308. 